\title{
Broadband Corbino spectroscopy and stripline resonators to study the microwave properties of superconductors
}

\author{
Marc Scheffler ${ }^{1}$, M. Maximilian Felger ${ }^{1}$, Markus Thiemann ${ }^{1}$, Daniel Hafner ${ }^{1}$, Katrin Schlegel ${ }^{1}$, \\ Martin Dressel ${ }^{1}$, Konstantin S. llin $^{2}$, Michael Siegel ${ }^{2}$, Silvia Seiro ${ }^{3}$, Christoph Geibel ${ }^{3}$, Frank Steglich ${ }^{3}$ \\ ${ }^{1}$ 1. Physikalisches Institut, Universität Stuttgart, Pfaffenwaldring 57, 70569 Stuttgart, Germany \\ ${ }^{2}$ Institut für Mikro- und Nanoelektronische Systeme (IMS), KIT, Hertzstraße 16, 76187 Karlsruhe, Germany \\ ${ }^{3}$ Max Planck Institute for Chemical Physics of Solids, Nöthnitzer Straße 40, 01187 Dresden, Germany
}

\begin{abstract}
Superconducting materials are of great interest both for the fundamental understanding of electrons in solids as well as for a range of different applications. Studying superconductors with microwaves offers a direct experimental access to the electrodynamic response of these materials, which in turn can reveal fundamental material properties such as the superconducting penetration depth. Here we describe two different techniques to study superconductors at microwave frequencies: the broadband Corbino approach can cover frequencies from the $\mathrm{MHz}$ range up to $50 \mathrm{GHz}$ continuously but is limited to thin-film samples whereas the stripline resonators are sensitive enough to study low-loss single crystals but reveal data only at a set of roughly equidistant resonant frequencies. We document the applicability of these two techniques with data taken on an ultrathin TaN film and a single crystal of the heavy-fermion superconductor $\mathrm{CeCu}_{2} \mathrm{Si}_{2}$, respectively.
\end{abstract}

\section{Section: RESEARCH PAPER}

Keywords: Superconductivity; microwaves; optical response; microwave spectroscopy

Citation: Marc Scheffler, M. Maximilian Felger, Markus Thiemann, Daniel Hafner, Katrin Schlegel, Martin Dressel, Konstantin S. Ilin, Michael Siegel, Silvia Seiro, Christoph Geibel, Frank Steglich, Broadband Corbino spectroscopy and stripline resonators to study the microwave properties of superconductors, Acta IMEKO, vol. 4, no. 3, article 8, September 2015, identifier: IMEKO-ACTA-04 (2015)-03-08

Editor: Paolo Carbone, University of Perugia, Italy

Received February 11, 2015; In final form April 9, 2015; Published September 2015

Copyright: (C) 2015 IMEKO. This is an open-access article distributed under the terms of the Creative Commons Attribution 3.0 License, which permits unrestricted use, distribution, and reproduction in any medium, provided the original author and source are credited

Funding: This work was supported by Deutsche Forschungsgemeinschaft (DFG)

Corresponding author: Marc Scheffler, e-mail: scheffl@pi1.physik-uni-stuttgart.de

\section{INTRODUCTION}

Superconductivity belongs to the most intensively studied phenomena of solid state physics. This research is driven both by the interest to gain fundamental understanding of electronic properties of solids and the aim to establish new applications of superconductors or to improve the existing ones. Both fields require experimental techniques to measure the characteristic material properties that characterize a superconductor. While certain "static" approaches like measuring the dc resistivity, the dc magnetization, or the specific heat are routine approaches in the material development and characterization of superconductors, electrodynamics experiments are much less common. In this contribution, we first motivate why microwave studies are particularly helpful in superconductivity research and then we present two complementary techniques, the broadband Corbino approach and the multi-frequency stripline resonators, that we have implemented successfully to study different types of superconductors using microwaves.

\subsection{Optical response of superconductors}

Though microwave experiments are technically performed in a very different manner compared to conventional optics, the actual outcome is rather similar: one probes the electrodynamics of the sample under study. Therefore, we briefly review the optical response of superconductors with a special focus on microwave frequencies [1], [2].

The crucial energy scale of the optical response of a superconductor is the superconducting energy gap $\Delta$ that in the case of a weak-coupling BCS superconductor is related to the critical temperature $T_{\mathrm{c}}$ via $2 \Delta=3.53 k_{\mathrm{B}} T_{\mathrm{c}}$. With typical $T_{\mathrm{c}}$ of the order of a few $K$, the energy gap is found in the range of 
$\mu \mathrm{eV}$ to $\mathrm{meV}$, i.e. up to $\mathrm{THz}$ frequencies. This means that at microwave frequencies one operates well within the superconducting energy gap and therefore probes the response of the superconducting ground state of the material.

At these frequencies, two types of charge carriers contribute to the electrodynamics: firstly, there are the Cooper pairs that constitute the superconducting condensate, the fundamental manifestation of superconductivity. Secondly, there are thermally excited quasiparticles that are lossy (i.e. Ohmic with respect to conduction) like conventional metallic electrons, but that do not show up in de resistance measurements because they are short-circuited by the loss-less Cooper pairs. Microwave measurements probe both kinds of electrons: the quasiparticles determine the real part $\sigma_{1}$ of the complex conductivity $\sigma=\sigma_{1}+i \sigma_{2}$ whereas the Cooper pairs dominate the imaginary part $\sigma_{2}$. In particular, $\sigma_{2}$ exhibits a $1 / \omega$ frequency dependence $(\omega=2 \pi f)$, which can be explained via KramersKronig transformation of the $\delta(\omega)$-peak of the Cooper pairs in $\sigma_{1}$. The magnitude of the $1 / \omega$ dependence directly depends on the Cooper pair density, which in turn is related to the superconducting penetration depth.

\subsection{General aspects of optical and microwave measurements}

Optical measurements are contact free and usually only demand a sample significantly bigger than the wavelength, i.e. typically of size of order $\mathrm{mm}$ and with at least one flat surface. Compared to other spectroscopic techniques, these requirements can often be met rather easily. However, in the case of conducting samples, the crucial question is whether the sensitivity of a particular optical technique is sufficient to resolve the features of interest. In particular for superconductors, this is a stringent restriction as the optical reflectivity of a bulk superconductor is very close to unity and therefore is extremely difficult to study even with state-of-theart optical spectrometers of the relevant spectral range, i.e. typically at $\mathrm{THz}$ and/or infrared frequencies. At microwave frequencies, the situation is in principle even more difficult, as the lower frequency of the employed radiation directly means reflectivity yet closer to unity. However, because of the rather long wavelength of microwave radiation, typically of order $\mathrm{cm}$, the employed experimental techniques do not follow optical routines, but are intrinsically different ones where the sample as well as the guides for the microwave radiation are smaller than the wave-length. I.e. although the fundamental physics of the microwave response of superconductors follows the theory of "optics of superconductors", the experiments are rather different than a conventional optics approach.

\section{MICROWAVE MEASUREMENTS ON SUPERCONDUCTORS}

A very well established and very versatile technique to study the microwave properties of conducting materials is cavity resonators [3]. Here, a three-dimensional conducting cavity has dimensions of order of the wavelength, and the sample under study acts as a small perturbation. The major disadvantages of this technique are that it is usually operated only at a single frequency (i.e. one does not gain any spectral information) and that it is difficult to obtain quantitative information due to the in general - poorly defined geometry (whereas relative information, e.g. as a function of temperature, is obtained easily).

Therefore, different new experimental techniques have been devised. These can be roughly divided into two classes: the first

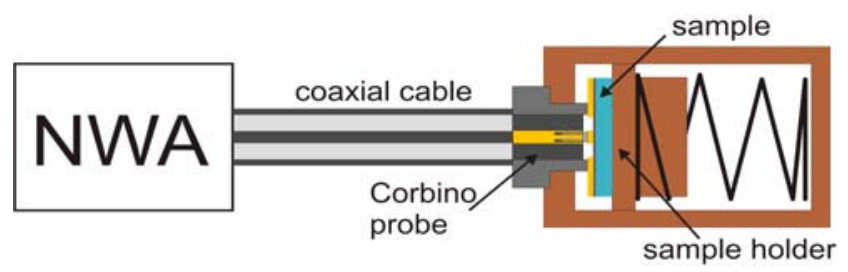

Figure 1. Scheme of a Corbino spectrometer. The network analyzer (NWA) measures the reflection coefficient of the microwave signal that travels in the coaxial cable and is reflected by the sample.

group comprises truly broadband techniques that cover the full frequency dependence. Here two approaches have been applied to the study of superconductors, namely the Corbino approach [4], [5] and the bolometric approach [6]. The main difference in their capabilities is that the Corbino approach reveals phase information (giving amplitude and phase of the response) but has a rather poor sensitivity, whereas the bolometric approach is very sensitive but does not reveal phase information. The second group is based on resonators that can be operated at several frequencies. While our approach below is based on onedimensional transmission line structures, namely superconducting stripline resonators [7], [8], a threedimensional resonator with a dielectric resonator in its core has recently also been established successfully [9].

\section{BROADBAND CORBINO SPECTROSCOPY}

In Corbino spectroscopy, the flat sample terminates the open end of a coaxial cable (schematically shown in Figure 1). In this way, the sample reflects the microwave signal traveling in the coaxial cable, and the reflection coefficient $S_{11}$, which can conveniently be measured with a network analyzer, then directly yields the impedance $Z_{\mathrm{L}}$ of the sample via the following relation (with $Z_{0}=50 \Omega$ the characteristic impedance of the coaxial cable):

$Z_{L}=Z_{0} \frac{1+S_{11}}{1-S_{11}}$

While this approach is commonly used at room temperature and in particular in the study of dielectrics [10], it is much more difficult for the case of superconductors. This is due to the elaborate calibration procedure that has to be applied to correct for the strongly temperature-dependent transmission properties of the coaxial cable [4], [5]. Different groups have come up with different schemes how this challenge can be met most successfully. It turns out that the best approach can differ drastically depending on the particular goal. In contrast to groups that concentrate on detailed studies of a single sample (or a selected few) [11], we have focused on a procedure that allows a high throughput combined with rather low temperatures. Presently, we operate a Corbino spectrometer with one cooldown per day, base temperature of $1.1 \mathrm{~K}$ (in a ${ }^{4} \mathrm{He}$ cryostat), frequency range $45 \mathrm{MHz}$ to $50 \mathrm{GHz}$, and two samples measured during each cooldown. With this rate, we can easily study extended sets of samples. Current examples are $\mathrm{NbN}$ and $\mathrm{TaN}$ thin films as a function of deposition parameters. Ultrathin films of these materials with thicknesses about $5 \mathrm{~nm}$ or even smaller are interesting both for applications in superconducting singlephoton detectors [12], [13] as well as for understanding of the so-called superconductor-to-insulator transition (SIT) [14], [15]. The typical critical temperatures of these materials are of order $5 \mathrm{~K}$ to $10 \mathrm{~K}$, and as a result the energy gap is located in the 


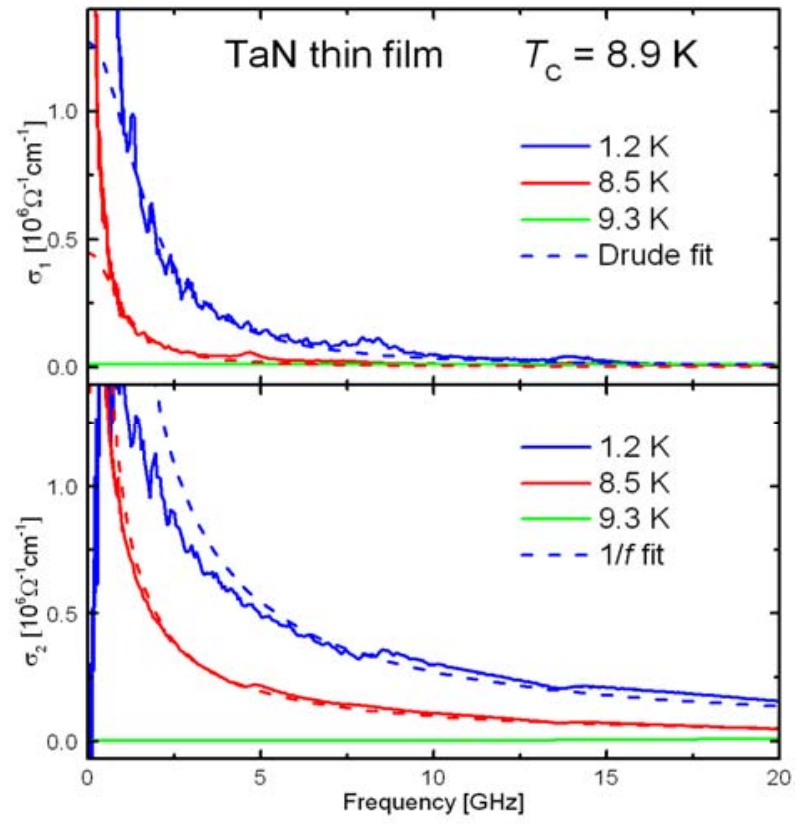

Figure 2. Microwave conductivity spectra of a TaN thin film, measured with a Corbino spectrometer.

$\mathrm{THz}$ frequency range, much higher than accessible with Corbino spectroscopy. However, for such ultrathin films, $\mathrm{THz}$ transmission measurements are easily possible and can directly determine the energy gap, whereas these studies usually have difficulties to resolve quasiparticle dynamics at the lowfrequency limit of their spectral range [1], [16]. Therefore, the combination of Corbino spectroscopy at $\mathrm{GHz}$ frequencies and $\mathrm{THz}$ transmission measurements is an ideal match to study the complete charge dynamics in superconductors, if the materials are available as very thin films.

If a conductive thin sample is studied in Corbino spectroscopy and if the microwave fields and current density are uniform through the sample thickness (which usually is the case for conductive thin films of thickness up to several tens of $\mathrm{nm})$, the complex conductivity of the sample can directly be calculated from the sample impedance $Z_{\mathrm{L}}$ as follows:

$\sigma=\frac{\ln \left(a_{2} / a_{1}\right)}{2 \pi d Z_{L}}$

Here $d$ is the thickness of the superconducting thin film and $a_{1}$ and $a_{2}$ are the inner and outer diameters of the Corbino disk, respectively [4], [5]. This Corbino disk is created by deposition of concentric conductive contacts (e.g. made of gold) onto the superconducting film, and it defines the geometry of the sample impedance $Z_{\mathrm{L}}$ that is probed. The thin-film samples are usually deposited onto dielectric substrates, and typically these substrates do not affect Corbino microwave measurements much in the frequency range below $20 \mathrm{GHz}$ [17], [18].

In Figure 2 we show typical microwave spectra, both real and imaginary parts of the conductivity, for a TaN film of thickness $5 \mathrm{~nm}$ that was sputtered onto a sapphire substrate. For temperatures above the critical temperature $T_{\mathrm{c}}=8.9 \mathrm{~K}$, the real part $\sigma_{1}$ is frequency independent and the imaginary part $\sigma_{2}$ basically vanishes, as expected for a conventional metal at $\mathrm{GHz}$ frequencies. Below $T_{\mathrm{c}}, \sigma_{1}$ rises strongly upon cooling. Our frequency range is well below the energy gap, and therefore $\sigma_{1}$ is not suppressed yet compared to the normal-state value even for the lowest temperature of $1.2 \mathrm{~K}$ [19]. The detailed frequency dependence reflects the density of states in the superconducting state and can phenomenologically be fitted with the Drude formula over a substantial frequency range [19]. Also $\sigma_{2}$ increases strongly upon cooling below $T_{\mathrm{c}}$, and its characteristic $1 / f$ frequency dependence gives direct access to the Cooper pair density and the penetration depth [1], [19]. Corbino spectroscopy at ${ }^{4} \mathrm{He}$ temperatures is thus well suited to study thin films of superconductors with $T_{\mathrm{c}}$ of $1.5 \mathrm{~K}$ and higher.

Considering that for many spectroscopic studies one desires the photon energy in a similar range as the thermal energy, we have also implemented a Corbino setup at ${ }^{3} \mathrm{He}$ temperatures [20]. Here the requirements for the calibration of the microwave coaxial cable in principle are even more stringent, but since in our laboratory we have a ${ }^{4} \mathrm{He}$ Corbino spectrometer well established, we limit our ${ }^{3} \mathrm{He}$ spectrometer to the temperature range $0.45 \mathrm{~K}$ to $2.0 \mathrm{~K}$, and we can use the overlap in temperature with the ${ }^{4} \mathrm{He}$ setup for additional calibration purposes.

All our studies mentioned so far, as well as Corbino experiments on superconductors by other groups [11], [19]-[24], are limited to thin-film samples (thickness of a few tens of nm or less). The reason is the limited sensitivity of Corbino spectroscopy [5]. But this is no restriction if the superconductor of interest can be prepared as thin films with the same material properties as the more commonly studied bulk samples or if the particular properties of interest only become manifest in thin films, like the SIT. For highly disordered superconductors close to the SIT, the conductivity in the superconducting state at frequencies below $2 \Delta$ has recently gained substantial attention because deviations from the canonical predictions of BCS theory might occur [25]-[28].

\section{SUPERCONDUCTING STRIPLINE RESONATORS}

Many unconventional superconductors, which are the topic of present research, are not available as thin film samples and as such not accessible with microwave Corbino spectroscopy. Many of these are ternary, quaternary, or even more complex intermetallics, and growth of high-quality thin films is extremely demanding [29]-[31]. Furthermore, $T_{\mathrm{c}}$ of unconventional superconductors is often suppressed if the mean free path of the conduction electrons is reduced, and then the small thickness of thin films can be an intrinsic limitation to superconductivity in these materials. To still be able to study these materials with microwaves as a function of frequency, we have developed a resonant technique for bulk samples that can be operated at numerous, roughly equidistant frequencies.

The general idea is that in a transmission line structure based on a stripline (also called triplate), as shown as cross section in Figure 3, one of the ground planes, which surround the signalcarrying center strip, can be formed by the conducting bulk material of interest. To be sensitive to low-loss samples, the stripline is created as a one-dimensional transmission line resonator, which has a fundamental frequency typically between $1 \mathrm{GHz}$ and $3 \mathrm{GHz}$ (depending on sample size) and which can easily be excited at numerous harmonics, i.e. one obtains a set of data at roughly equidistant frequencies, spanning a range up to approximately $15 \mathrm{GHz}$ at present. Particular care has to be taken in the choice of the center conductor material: here, the density of the microwave currents is an order of magnitude larger than in the ground planes, and therefore the overall 


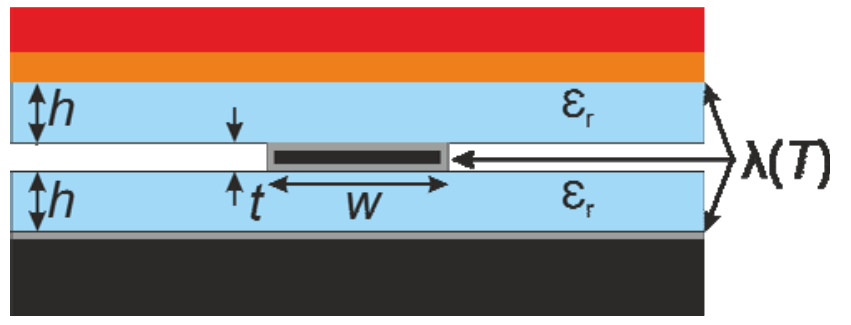

Figure 3. Schematic cross section of a stripline as used in resonator studies. The signal-carrying center strip (thickness $t=1 \mu \mathrm{m}$, width $w=45 \mu \mathrm{m}$ ) is sandwiched between two ground planes at distance $h=127 \mu \mathrm{m}$ (thickness of dielectric spacers, made of sapphire, with dielectric constant $\varepsilon_{\mathrm{r}}$ ). Center strip and lower ground plane are made of a low-loss superconductor $(\mathrm{Pb}$ or $\mathrm{Nb}$ ), whereas the bulk superconducting sample of interest acts as upper ground plane of the stripline. The microwave fields enter the superconductors within the superconducting penetration depth $\lambda$ (indicated as shaded regions).

losses of the stripline first of all depend on the center conductor. If we want to study a superconductor as ground plane, this means that the center conductor has to be fabricated from a thin film of an even "better" superconductor, i.e. one with even lower microwave losses at low temperatures. In general, such very low losses can be achieved if the resonator is operated at temperatures well below $T_{\mathrm{c}}$, i.e. materials with a high $T_{\mathrm{c}}$ are desirable for the center conductor. However, the materials should be conventional s-wave superconductors, as superconductors with nodes have much higher losses at low temperatures due to the larger amount of thermally excited quasiparticles. This restriction excludes the high- $T_{\mathrm{c}}$ cuprate superconductors, which are $d$-wave, from this application. Therefore, we fabricate our stripline resonators either from $\mathrm{Nb}$ or $\mathrm{Pb}$ thin films [8], [32].

While we originally implemented this technique to study unconventional metals, such as heavy fermions with their peculiar microwave properties [33]-[39], it turned out that this technique is also well capable to study bulk superconductors if their $T_{\mathrm{c}}$ is lower than that of the stripline material [8]. After test measurements on the conventional elemental superconductors $\mathrm{Ta}$ and $\mathrm{Sn}$ [8], we now apply it to the unconventional heavyfermion superconductor $\mathrm{CeCu}_{2} \mathrm{Si}_{2}$ [40]. While $\mathrm{CeCu}_{2} \mathrm{Si}_{2}$ was long believed to be a $d$-wave superconductor [41], recent studies instead suggest that it is a two-gap s-wave superconductor [42]. This question is of particular interest as it is related to the possible pairing mechanism for superconductivity, which is thought to be of magnetic nature in $\mathrm{CeCu}_{2} \mathrm{Si}_{2}$ [43]. One possibility to address whether a superconductor has an order parameter with nodes or not is measure the temperature dependence of its penetration depth. This can be done with microwaves.

So far, no microwave studies on $\mathrm{CeCu}_{2} \mathrm{Si}_{2}$ have been reported. One difficulty here is the rather low $T_{\mathrm{c}}$ of around $0.6 \mathrm{~K}$. While this temperature can be reached with ${ }^{3} \mathrm{He}$ cryostats, a detailed study of the penetration depth requires a base temperature of order $T_{c} / 10$ or lower, i.e. one has to employ a ${ }^{3} \mathrm{He} /{ }^{4} \mathrm{He}$ dilution refrigerator. Here, another advantage of our stripline probe comes into play: its physical dimensions are much smaller than the fundamental wavelength, as this onedimensional resonator can be shaped as a meander. As a result, the typical mounting of resonator and sample amounts to just a few $\mathrm{cm}^{3}$, a volume that can easily be incorporated in a commercial dilution refrigerator. Furthermore, the microwave signals are guided from the room-temperature electronics to the

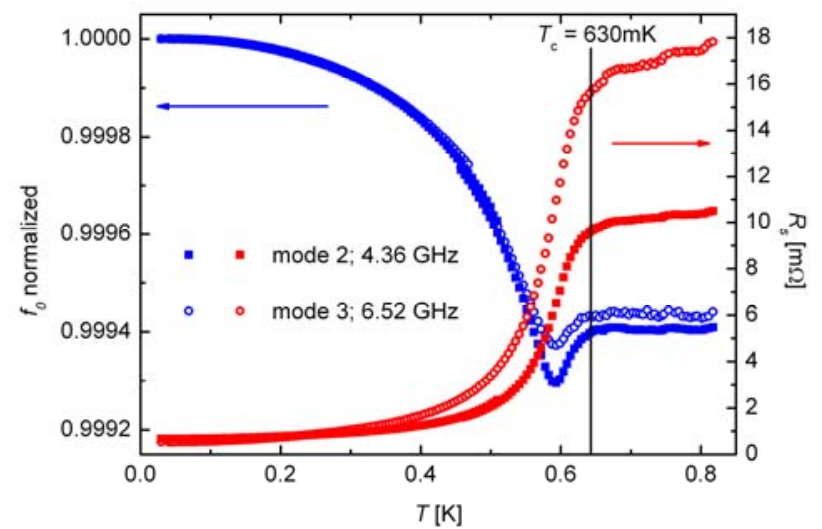

Figure 4. Temperature dependence of stripline resonator frequency $f_{0}$ (normalized to the frequency at the lowest temperature) and sample surface resistance $R_{\mathrm{s}}$ measured on a $\mathrm{CeCu}_{2} \mathrm{Si}_{2}$ single crystal sample. Both quantities are shown for two different resonator modes.

cryogenic probe and back via coaxial cables, which is much more generic than waveguides that are often used for threedimensional cavity resonators. Therefore, the stripline resonator is a widely applicable, very sensitive technique to study the microwave response of superconductors. In contrast to many other resonator approaches, here it is rather simple to obtain data at several different frequencies, i.e. spectral information. While for addressing the penetration depth, which in this range basically is frequency independent, a single resonator frequency might be sufficient, there are other questions of interest, such as the quasiparticle dynamics, where data as a function of frequency are crucially required. These frequency dependences are usually broad, and therefore the rather restricted spectral resolution of order $1 \mathrm{GHz}$ is sufficient, whereas the truly broadband Corbino approach has a much better frequency resolution that can also reveal narrow spectral structures.

As a demonstration for $\mathrm{mK}$ measurements using a stripline resonator, in Figure 4 we show data obtained on a $\mathrm{CeCu}_{2} \mathrm{Si}_{2}$ single crystal for two resonator modes, at frequencies $4.36 \mathrm{GHz}$ and $6.52 \mathrm{GHz}$, measured during the same temperature sweep. When cooled below $T_{c}$, the surface resistance $R_{s}$ decreases strongly due to the reduction of microwave losses. At the same time, the resonator frequency $f_{0}$ increases because of the decreasing penetration depth. Above $T_{\mathrm{c}}$, in the metallic heavyfermion state, $R_{\mathrm{s}}$ is also temperature dependent, even for these low temperatures below $1 \mathrm{~K}$. This indicates that the electronic conduction is governed by intrinsic scattering mechanisms (in contrast to temperature-independent impurity scattering), and it shows promise for future studies of the microwave response of heavy fermions in the normal state of $\mathrm{CeCu}_{2} \mathrm{Si}_{2}$, an interesting field of study on its own [33]-[38]. Comparing the two resonator frequencies reveals that the superconducting penetration depth well below $T_{\mathrm{c}}$ does not depend on frequency, whereas $R_{\mathrm{s}}$ is noticeably enhanced for the higher frequency, both below and above $T_{\mathrm{c}}$, as expected.

Our stripline approach thus can reveal the frequencydependent microwave response of a superconductor. In the present case of $\mathrm{CeCu}_{2} \mathrm{Si}_{2}$, where a ${ }^{3} \mathrm{He} /{ }^{4} \mathrm{He}$ dilution refrigerator was employed, measurements down to $30 \mathrm{mK}$ were performed. Thus a wide range of unconventional low- $T_{\mathrm{c}}$ superconductors can be studied with this technique. Recently, another microwave approach, namely a multimode dielectric resonator [9], was used to study the heavy-fermion superconductor CeCoIn 5 [44]. These two techniques have rather complementary 
advantages: our stripline approach offers a wide and flexible range of numerous $\mathrm{GHz}$ frequencies, it can be used to resolve anisotropy in the microwave response, and due to its compact design it can conveniently be installed into a wide range of dilution refrigerators (thus achieving even lower temperatures than those demonstrated in [44]), whereas the multimode dielectric resonator can be operated in high magnetic field, which is not possible for our stripline approach based on the superconductors $\mathrm{Pb}$ or $\mathrm{Nb}$.

\section{CONCLUSIONS}

We have discussed why microwave measurements on superconductors are powerful tools to understand their electrodynamics and to obtain detailed information about fundamental characteristics of superconductivity, such as the temperature-dependent penetration depth. In recent years, several groups have developed new techniques for cryogenic microwave spectroscopy. While the Corbino approach is particularly popular and offers the possibility to cover a very large frequency range completely, it is limited to the study of very thin films. As an alternative, we have established a rather different technique, which are stripline resonators where the bulk sample of interest is one ground plane of the stripline. With these two approaches available, we can cover a very large range of superconducting materials and address their microwave properties both for fundamental research as well as for materials optimization for applications.

\section{ACKNOWLEDGEMENT}

We thank G. Untereiner for assistance in probe and sample preparation and U. Pracht and L. Benfatto for helpful discussions.

\section{REFERENCES}

[1] U.S. Pracht, E. Heintze, C. Clauss, D. Hafner, R. Bek, D. Werner, S. Gelhorn, M. Scheffler, M. Dressel, D. Sherman, B. Gorshunov, K.S. Il'in, D. Henrich, M. Siegel, Electrodynamics of the superconducting state in ultra-thin Films at $\mathrm{THz}$ frequencies, IEEE Trans. THz Sci. Technol. 3 (2013) pp. 269-280.

[2] M. Dressel, Electrodynamics of metallic superconductors, Adv. Condens. Matter Phys. 2013 (2013) article 104379.

[3] O. Klein, S. Donovan, M. Dressel, G. Grüner, Microwave cavity perturbation technique: part I: principles, Int. J. Infrared Millim. Waves 14 (1993) pp. 2423-2457; S. Donovan, O. Klein, M. Dressel, K. Holczer, G. Grüner, Microwave cavity perturbation technique: part II: experimental scheme, Int. J. Infrared Millim. Waves 14 (1993) pp. 2459-2487; M. Dressel, O. Klein, S. Donovan, G. Grüner, Microwave cavity perturbation technique: part III: applications, Int. J. Infrared Millim. Waves 14 (1993) pp. 2489-2517.

[4] J.C. Booth, D.H. Wu, S.M. Anlage, A broadband method for the measurement of the surface impedance of thin films at microwave frequencies, Rev. Sci. Instrum. 65 (1994) pp. 20822090.

[5] M. Scheffler, M. Dressel, Broadband microwave spectroscopy in Corbino geometry for temperatures down to $1.7 \mathrm{~K}$, Rev. Sci. Instrum. 76 (2005) article 074702.

[6] P.J. Turner, D.M. Broun, S. Kamal, M.E. Hayden, J.S. Bobowski, R. Harris, D.C. Morgan, J.S. Preston, D.A. Bonn, W.N. Hardy, Bolometric technique for high-resolution broadband microwave spectroscopy of ultra-low-loss samples, Rev. Sci. Instrum. 75 (2004) pp. 124-135.

[7] M.S. DiIorio, A.C. Anderson, B.Y. Tsaur, $\mathrm{rf}$ surface resistance of Y-Ba-Cu-O thin films, Phys. Rev. B. 38 (1988) pp. 7019-7022.
[8] D. Hafner, M. Dressel, M. Scheffler, Surface-resistance measurements using superconducting stripline resonators, Rev. Sci. Instrum. 85 (2014) article 014702.

[9] W.A. Huttema, B. Morgan, P.J. Turner, W.N. Hardy, X. Zhou, D.A. Bonn, R. Liang, D.M. Broun, Apparatus for high-resolution microwave spectroscopy in strong magnetic fields, Rev. Sci. Instrum. 77 (2006) article 023901.

[10] J. Krupka, Frequency domain complex permittivity measurements at microwave frequencies, Meas. Sci. Technol. 17 (2006) pp. R55-R70.

[11] W. Liu, M. Kim, G. Sambandamurthy, N.P. Armitage, Dynamical study of phase fluctuations and their critical slowing down in amorphous superconducting films, Phys. Rev. B 84 (2011) article 024511.

[12] A. Engel, A. Aeschbacher, K. Inderbitzin, A. Schilling, K. Il'in, M. Hofherr, M. Siegel, A. Semenov, H.-W. Hübers, Tantalum nitride superconducting single-photon detectors with low cut-off energy, Appl. Phys. Lett. 100 (2012) article 062601.

[13] D. Henrich, S. Dörner, M. Hofherr, K. Il'in, A. Semenov, E. Heintze, M. Scheffler, M. Dressel, M. Siegel, Broadening of hotspot response spectrum of superconducting $\mathrm{NbN}$ nanowire single-photon detector with reduced nitrogen content, J. Appl. Phys. 112 (2012) article 074511.

[14] V.F. Gantmakher, V.T. Dolgopolov, Superconductor-insulator quantum phase transition, Phys. Usp. 53 (2010) pp. 1-49.

[15] J. Yong, T.R. Lemberger, L. Benfatto, K. Ilin, M. Siegel, Robustness of the Berezinskii-Kosterlitz-Thouless transition in ultrathin $\mathrm{NbN}$ films near the superconductor-insulator transition, Phys. Rev. B 87 (2013) article 184505.

[16] U.S. Pracht, M. Scheffler, M. Dressel, D.F. Kalok, C. Strunk, T.I. Baturina, Direct observation of the superconducting gap in a thin film of titanium nitride using terahertz spectroscopy, Phys. Rev. B 86 (2012) article 184503.

[17] H. Kitano, T. Ohashi, A. Maeda, Broadband method for precise microwave spectroscopy of superconducting thin films near the critical temperature, Rev. Sci. Instrum. 79 (2008) article 074701.

[18] M.M. Felger, M. Dressel, M. Scheffler, Microwave resonances in dielectric samples probed in Corbino geometry: simulation and experiment, Rev. Sci. Instrum. 84 (2013) article 114703.

[19] K. Steinberg, M. Scheffler, M. Dressel, Quasiparticle response of superconducting aluminum to electromagnetic radiation, Phys. Rev. B 77 (2008) article 214517.

[20] K. Steinberg, M. Scheffler, M. Dressel, Broadband microwave spectroscopy in Corbino geometry at ${ }^{3} \mathrm{He}$ temperatures, Rev. Sci. Instrum. 83 (2012) article 024704.

[21] D.H. Wu, J.C. Booth, S.M. Anlage, Frequency and field variation of vortex dynamics in $\mathrm{YBa}_{2} \mathrm{Cu}_{3} \mathrm{O}_{7-\delta}$, Phys. Rev. Lett. 75 (1995) pp. 525-528.

[22] H. Kitano, T. Ohashi, A. Maeda, I. Tsukada, Critical microwaveconductivity fluctuations across the phase diagram of superconducting $\mathrm{La}_{2-} \mathrm{Sr}_{\mathrm{x}} \mathrm{CuO}_{4}$ thin films, Phys. Rev. B 73 (2006) article 092504.

[23] E. Silva, N. Pompeo, S. Sarti, Wideband microwave measurements in $\mathrm{Nb} / \mathrm{Pd}_{84} \mathrm{Ni}_{16} / \mathrm{Nb}$ structures and comparison with thin Nb films, Supercond. Sci. Technol. 24 (2011) article 024018.

[24] M. Mondal, A. Kamlapure, S.C. Ganguli, J. Jesudasan, V. Bagwe, L. Benfatto, P. Raychaudhuri, Enhancement of the finitefrequency superfluid response in the pseudogap regime of strongly disordered superconducting films, Sci. Rep. 3 (2013) article 1357.

[25] S. Gazit, D. Podolsky, A. Auerbach, D. P. Arovas, Dynamics and conductivity near quantum criticality, Phys. Rev. B 88 (2013) article 235108.

[26] M. Swanson, Y.L. Loh, M. Randeria, N. Trivedi, Dynamical conductivity across the disorder-tuned superconductor-insulator transition, Phys. Rev. X 4 (2014) article 021007.

[27] T. Cea, D. Bucheli, G. Seibold, L. Benfatto, J. Lorenzana, C. Castellani, Optical excitation of phase modes in strongly disordered superconductors, Phys. Rev. B 89 (2014) article 174506. 
[28] D. Sherman, U.S. Pracht, B. Gorshunov, S. Poran, J. Jesudasan, M. Chand, P. Raychaudhuri, M. Swanson, N. Trivedi, A. Auerbach, M. Scheffler, A. Frydman, M. Dressel, The Higgs mode in disordered superconductors close to a quantum phase transition, Nature Phys. 11 (2015) pp. 188-192.

[29] M. Jourdan, A. Zakharov, M. Foerster, H. Adrian, Evidence for multiband superconductivity in the heavy fermion compound $\mathrm{UNi}_{2} \mathrm{Al}_{3}$, Phys. Rev. Lett. 91, No.9 (2004) article 097001.

[30] Y. Mizukami, H. Shishido, T. Shibauchi, M. Shimozawa, S. Yasumoto, D. Watanabe, M. Yamashita, H. Ikeda, T. Terashima, H. Kontani, Y. Matsuda, Extremely strong-coupling superconductivity in artificial two-dimensional Kondo lattices, Nat. Phys. 7 (2011) pp. 849-853.

[31] M. Scheffler, T. Weig, M. Dressel, H. Shishido, Y. Mizukami, T. Terashima, T. Shibauchi, Y. Matsuda, Terahertz conductivity of the heavy-fermion state in CeCoIn, J. Phys. Soc. Jpn. 82 (2013) article 043712

[32] M. Thiemann, D. Bothner, D. Koelle, R. Kleiner, M. Dressel, M. Scheffler, Niobium stripline resonators for microwave studies on superconductors, J. Phys.: Conf. Ser. 568 (2014) article 022043.

[33] L. Degiorgi, The electrodynamic response of heavy-electron compounds, Rev. Mod. Phys. 71 (1999) pp. 687-734.

[34] M. Scheffler, M. Dressel, M. Jourdan, H. Adrian, Extremely slow Drude relaxation of correlated electrons, Nature 438 (2005) pp. 1135-1137.

[35] M. Scheffler, M. Dressel, M. Jourdan, H. Adrian, Dynamics of heavy fermions: Drude response in $\mathrm{UPd}_{2} \mathrm{Al}_{3}$ and $\mathrm{UNi}_{2} \mathrm{Al}_{3}$, Physica B 378-380 (2006) pp. 993-994.

[36] M. Scheffler, M. Dressel, M. Jourdan, Microwave conductivity of heavy fermions in $\mathrm{UPd}_{2} \mathrm{Al}_{3}$, Eur. Phys. J. B 74 (2010) pp. 331338.
[37] D.N. Basov, R.D. Averitt, D.v.d. Marel, M. Dressel, K. Haule, Electrodynamics of correlated electron materials, Rev. Mod. Phys. 83 (2011) pp.471-541.

[38] M. Scheffler, K. Schlegel, C. Clauss, D. Hafner, C. Fella, M. Dressel, M. Jourdan, J. Sichelschmidt, C. Krellner, C. Geibel, F. Steglich, Microwave spectroscopy on heavy-fermion systems: probing the dynamics of charges and magnetic moments, Phys. Status Solidi B 250 (2013) pp.439-449.

[39] D. Hafner, M. Dressel, O. Stockert, K. Grube, H.v. Löhneysen, M. Scheffler, Anomalous microwave surface resistance of $\mathrm{CeCu}_{6}$, JPS Conf. Proc. 3 (2014) article 012016.

[40] F. Steglich, J. Aarts, C.D. Bredl, W. Lieke, D. Meschede, W. Franz, H. Schäfer, Superconductivity in the presence of strong Pauli paramagnetism: $\mathrm{CeCu}_{2} \mathrm{Si}_{2}$, Phys. Rev. Lett. 43 (1979) pp. 1892-1896.

[41] F. Steglich, J. Arndt, O. Stockert, S. Friedemann, M. Brando. C. Klingner, C. Krellner, C. Geibel, S. Wirth, S. Kirchner, Q. Si, Magnetism, f-electron localization and superconductivity in 122type heavy-fermion metals, J. Phys. Condens. Matter 29 (2012) article 294201.

[42] S. Kittaka, Y. Aoki, Y. Shimura, T. Sakakibara, S. Seiro, C. Geibel, F. Steglich, H. Ikeda, K. Machida, Multiband superconductivity with unexpected deficiency of nodal quasiparticles in $\mathrm{CeCu}_{2} \mathrm{Si}_{2}$, Phys. Rev. Lett. 112 (2014) article 067002 .

[43] O. Stockert, J. Arndt, E. Faulhaber, C. Geibel, H.S. Jeevan, S. Kirchner, M. Loewenhaupt, K. Schmalzl, W. Schmidt, Q. Si, F. Steglich, Magnetically driven superconductivity in $\mathrm{CeCu}_{2} \mathrm{Si}_{2}$, Nat. Phys. 7 (2011) pp. 119-124.

[44] C.J.S. Truncik, W.A. Huttema, P.J. Turner, S. Özcan, N.C. Murphy, P.R. Carrière, E. Thewalt, K.J. Morse, A.J. Koenig, J.L. Sarrao, D.M. Broun, Nodal quasiparticle dynamics in the heavy fermion superconductor $\mathrm{CeCoIn}_{5}$ revealed by precision microwave spectroscopy, Nat. Commun. 4 (2013) article 2477. 